\title{
Relationship between static bending and compressive behaviour of particle-reinforced cement composites
}

\author{
A. Arias ${ }^{\mathrm{a}, *}$, P. Forquin ${ }^{\mathrm{b}}$, R. Zaera ${ }^{\mathrm{a}}$, C. Navarro ${ }^{\mathrm{a}}$ \\ a Department of Continuum Mechanics and Structural Analysis, University Carlos III of Madrid, Avda. de la Universidad 30, 28911 Leganés, Madrid, Spain \\ ${ }^{\mathrm{b}}$ Laboratory of Physics and Mechanics of Materials, UMR CNRS 75-54, University of Metz, Ile du Saulcy, 57045 Metz cedex, France
}

\begin{abstract}
A B S T R A C T
Innovative particle reinforced materials made of alumina particles and cement based matrix were designed, manufactured and tested to evaluate the potential use of ceramic aggregates in concretes. These particle reinforced composites were tested in three point bending and uniaxial compression con ditions to determine the influence of the shape and size of the ceramic inclusions, and the addition of sil ica fume on the mechanical properties. A specific methodology combining post mortem observations with a statistical analysis of tensile failure stresses (average strength and Weibull modulus) was con ducted to deduce the origin of failure for each cement based composite (porosity or ceramic particles/ matrix decohesion). A remarkable correlation is observed between bending failure stress level and the average strength measured under uniaxial compression loading. As main conclusion, addition of alumina particles in a mortar appears to strengthen or to weaken the composite depending on whether silica fume is used in the cementitious matrix.
\end{abstract}

\section{Introduction}

For a few decades, ceramic composites have been used to build protective systems which provide ballistic efficiency against the impact of low and medium calibre projectiles. For example, bilay ered armours, made of ceramic tiles as front plate and a ductile material (steel, aluminium alloy, fibre reinforced composite or a polymeric material) as backing plate, have been successfully em ployed $[1,2]$ to improve the impact behaviour of protections (and to decrease their weight). The front layer (e.g., ceramic) aims at breaking or blunting the hard core of the projectile and spreading out the energy of impact by forming a cone shaped cracking zone. The backing plate allows absorption of the residual kinetic energy of the debris (projectile and ceramic fragments). The high compres sive strength and the low density of ceramic materials make them particularly attractive as front layer material [3]. However, dynamic tensile stress fields develop within the target during impact leading to an intense damage of the ceramic made of numerous oriented micro cracks $[4,5]$. This fragmentation decreases greatly the resid ual strength of the front face and may significantly reduce the mul ti hit capability of the armour. One route imagined to avoid the motion of fragments resulting from a first impact is to use porous ceramic infiltrated by an aluminium alloy [5]. Another route con sists in reducing the extension of damage of the front face. This goal may be reached if a composite material made with a polymeric ma

\footnotetext{
* Corresponding author. Tel.: +34 916249161; fax: +34 916249430.

E-mail address: aariash@ing.uc3m.es (A. Arias).
}

trix and containing a high density of ceramic particles is used as front layer material [6 9 ]. In this work, cement composites were designed with a similar perspective, by incorporating high strength particles to improve the impact resistance of concrete materials [10] that present low cost and the ease of moulding.

Many authors have studied in the past the relation between the composition of cement composites made of aggregates and their strength under tensile and compression loadings [11 22]. In this way, an improvement can be made by careful selection, mixing and treatment of conventional components. The effect of adding silica fume and of the amount of water on the static strength and workability of high performance concrete has been investigated with extension [11 16]. These studies concluded that within the investigated range of the water/cement ratio $(0.251 .17)$, the smal ler the amount of water the higher the strength of concrete with out an addition of silica fume. However, it is necessary to use a super plasticizer at least in the low water/cement ratio $[11,12]$ to get constant workability of the concrete. Duval and Kadri [11] also show that the optimal super plasticizer dosage (for a constant workability) is minimum when the quantity of cement is nine times the quantity of silica fume. For higher or lower amounts of silica fume, the superplasticizer proportion has to be increased. According to Prokopski and Langier [12], a small amount of water influences favourably the critical stress intensity factor of concrete. Papadakis [13] obtained an optimal ratio closer to $15 \%$ to get the maximal compressive strength while Prokopski and Langier [12] confirm the beneficial effect of silica fume on the compressive strength or the critical stress intensity factor. Kearsley and 
Wainwright [14] used additive fills (fly ash or pozzolanic fill) in a high proportion (75\%) and they concluded that the fill does not re duce the strength when its dosage is smaller than that of cement. Bhanja and Sengupta [15] and Atis et al. [16] investigate many grades of concrete with different amounts of silica fume and water. Bhanja and Sengupta [15] obtained a significant improvement in the compressive and tensile (split and flexural) strengths of con cretes made with ordinary Portland cement when silica fume is incorporated; the optimum replacement percentage was not a con stant but depends on the water cementitius material ratio and on the property to be improved. The numerous tests performed by Atis et al. [16] indicate that a silica fume/cement ratio of $20 \%$ may be better for a similar water/cement ratio (0.3).

Moreover, several authors investigated recently the influence of the roughness and type of aggregates on the interfacial bond prop erties between them and mortar matrices. Caliskan [17] performed push out tests on three types of cylindrical aggregates (sandstone, limestone, granite aggregates) to determine experimentally the interfacial shear strength and he found that $20 \%$ silica fume replace ment of cement provided a better bonding effect than that in plain cement mortar, regardless of aggregate diameter and type. Rao and Prasad [18] showed how the roughness of the aggregate surface sig nificantly increases the tensile and shear bond strengths of the interface as a result of a better physical interaction. The experimen tal works of Li et al. [19] and Jo et al. [20] analysed the mechanical properties of nano cement mortars and their study shows that the compressive and flexural strengths of cement mortars mixed with nano particles are higher than that of plain cement mortar. An improvement of confined compressive strength can also be made by optimising the particles size distribution, reducing the water content and the maximum grain size of concretes [21,22].

During the impact of a projectile against a brittle material, a wide spectrum of damage is produced: cone cracking, spalling, cracks on proximal face and distal face, scabbing, and fragmenta tion [23] as a consequence of the tensile loadings that develop in the target. At the same time, compressive stresses develop in front of the projectile and thus the ballistic performance of concretes and ceramic materials have been related to their uniaxial compres sion strength [24 27]. Therefore, bending tests and uniaxial com pression tests are prerequisite prior to ballistic experiments on cement based composites.

Here we present the performance of new particle reinforced ce ment based composites with alumina aggregates of distinct sizes and shapes (angular and spherical particles) and with two types of cementitious matrices (with and without silica fume). The ratio of silica fume/cement used is $10 \%$ for a water/cement ratio equal to $41 \%$, close to the optimal values of previous studies of cement composites $[11,13,16]$. The aims of this work are to measure the ba sic static mechanical properties of these composite materials (as a prior step before determining the dynamic ones), to understand how their microstructure (types of alumina particles and of matrix) influences their strength. Therefore, different specimens were tested under flexural and uniaxial compression conditions. An intensive post mortem analysis of the specimens made it possible to relate qualitatively the differences observed in average and dis persion strengths for each composite with their microstructure.

\section{Manufacturing, microstructure and density of the composites}

\subsection{Selection of the components and manufacture}

The composite materials prepared in the course of this study use a cementitious matrix and four types of alumina particles. The ma trix is a self consolidating mortar of fine sand of quartz (Sifraco) and cement (52.5 HTS, Lafarge), with or without silica fume (Inasa). The admixture is the adjuvant Dynamon SP3 of Mapei.

To study the effect of the shape of the particles on cohesion be tween the matrix and the particles, two forms of alumina particles were used, one angular (Alcoa) with Vickers hardness HV $=1100$, obtained by sintering and subsequent crushing, and the other spherical (Caslab), sintered, with Vickers hardness HV $=1000$. Sur face of the alumina particles was extensively washed with high pressure water to improve mortar/aggregate bond.

The concrete grades were also manufactured keeping in mind the interest of performing impact tests with typical FSP (fragment simulating projectile) of $5.3 \mathrm{~mm}$ diameter and $1.3 \mathrm{~g}$ mass. Three types of angular particles (of 13,36 and $510 \mathrm{~mm}$ ) were used, which offers the advantage of being slightly smaller and bigger than the characteristic size of the projectile and one type of spher ical particles ( $4 \mathrm{~mm}$ diameter). All particles are well below that of the diameter of the compression specimens $(30 \mathrm{~mm})$ or of the 3 point bending specimens $(20 \mathrm{~mm}$ height, $15 \mathrm{~mm}$ width and $100 \mathrm{~mm}$ length). The concrete pastes of composite were prepared with a large capacity mixing machine $(40 \mathrm{l}$ ) and poured into ply wood moulds. Mortars are self consolidating and were stored in airtight container at room temperature (28 days).

Table 1

Nomenclature, composition and densities of cement-based composites

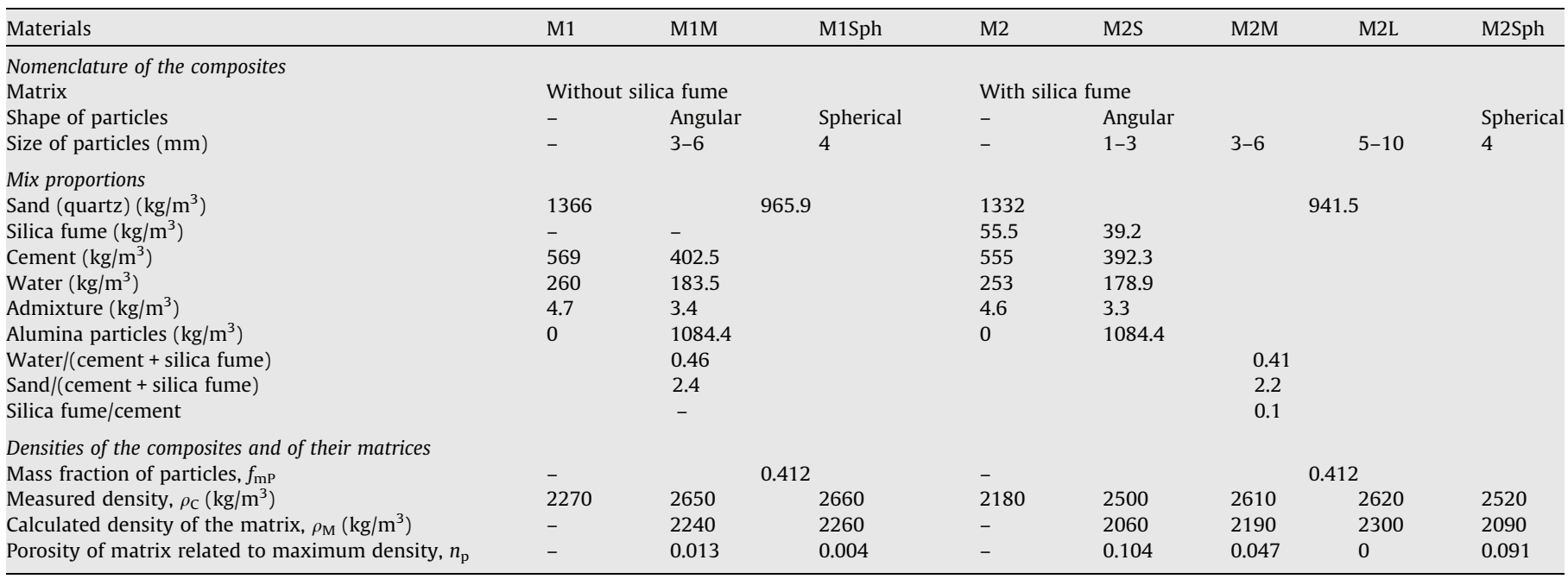




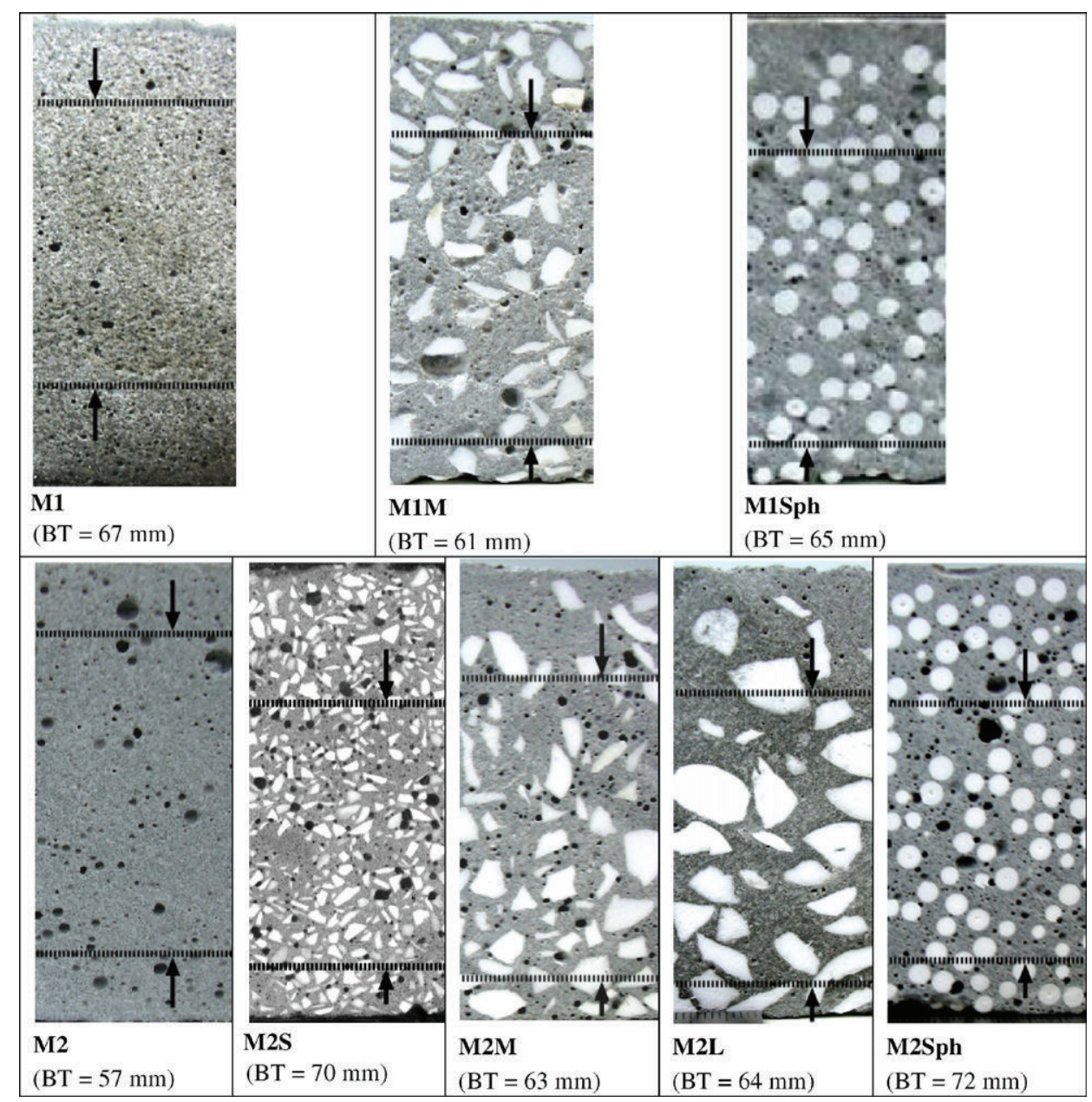

Fig. 1. Microstructure of the cement-based composites. Portion of the blocks used for specimens: $40 \mathrm{~mm}$ (BT: block thickness).

\subsection{Mix proportions and microstructure of the components}

To establish an easy to follow rule for the name of the con cretes considered in this study, the nomenclature shown in Table 1 was adopted ( $\mathrm{S}$ : small size particles, M: medium size particles, L: large size particles, Sph: spherical particles). Table 1 shows also the mix proportions of cement based composites with and without alumina particles. The roughly $30 \%$ volumetric fraction of the cera mic particles gives a total mass fraction of $41.2 \%$, chosen for the two formulae M1 and M2. In addition, the microstructure of each concrete is shown in Fig. 1. The absence of segregation is clearly observed in the portion of the blocks used for specimens $(40 \mathrm{~mm})$.

\subsection{Density of composites}

The density of the different mixtures was measured to deter mine the average density of the matrix of each concrete and to compare the equivalent porosity of mortars with or without parti cles. Volumes of $200 \mathrm{~cm}^{3}$, cut from the middle of the blocks (Fig. 1), were used, assumed to have the same characteristics as those used in the compression and bending tests of the study. The composites were dried at temperature of $90^{\circ} \mathrm{C}$ in climatic chamber during $24 \mathrm{~h}$ to eliminate residual water and then weighed with an error below $0.25 \%$. After, their volume was then calculated by measurement of the water displaced. Two different specimens of each batch were tested, and the difference between their densities was always less than $2 \%$. The weight of the same specimens was also determined after their immersion in water for $48 \mathrm{~h}$. It was found to have in creased by about $35 \%$ in comparison with the dry concretes $(+5 \%$ for mortars $\mathrm{M} 1$ and $\mathrm{M} 2,+3 \%$ for concretes with particles M1M, M1Sph, M2S, M2M, M2Sph, $+2.3 \%$ with M2L concretes). From the results of the density of the dried concretes and those of the ceramic particles, the density of their matrix $\left(\rho_{\mathrm{M}}\right)$ is deduced from Eq. (1):

$\rho_{\mathrm{M}} \quad\left(\begin{array}{ll}1 & f_{\mathrm{mP}}\end{array}\right)\left[\begin{array}{ccc}\rho_{\mathrm{C}} & \rho_{\mathrm{P}} \\ \hline \rho_{\mathrm{P}} & f_{\mathrm{mP}} & \rho_{\mathrm{C}}\end{array}\right]$,

in which $\rho_{\mathrm{P}}$ is the measured density of the particles (3.60 for angu lar and $3.56 \mathrm{~g} / \mathrm{cm}^{3}$ for spherical), $f_{\mathrm{mP}}$ is the mass fraction of the par ticles and $\rho_{\mathrm{C}}$ is the measured density of the concretes. Table 1 shows these values for the dry specimens.

The density of the particles (3.56 and $3.60 \mathrm{~g} / \mathrm{cm}^{3}$ ) appears slightly lower than that of sintered alumina $\left(3.74 \mathrm{~g} / \mathrm{cm}^{3},[27]\right)$. The density of the particles free M1 (without silica fume) is slightly higher than that of the M2 that contains silica fume, but this is not so surprising in view of Fig. 1 where the millimetric porosity is more or less absent in the M1 mortar but clearly present in the type M2. This confirms that even though silica fume may reduce the micro scopic porosity (values of size pore minor than $100 \mu \mathrm{m}$ ), it may in crease at the same time the millimetric porosity if the amount of water is not much changed. Influence of silica fume on the total porosity of concretes was mentioned previously by other workers $[28,29]$. The great amount of millimetric porosity within the M2 mortar may be due to the fact that there is no vibration during 
the processing. Since the concretes containing ceramic particles were not vibrated to avoid segregation (due to the higher density of the particles), the mortars also were not vibrated.

Again the density $\rho_{\mathrm{M}}$ for the particles loaded concretes with matrix M1 is clearly higher than that of the loaded M2 (Table 1). The density of the M1Sph matrix with spherical particles is above that of the M2Sph (2.26 against $2.09 \mathrm{~g} / \mathrm{cm}^{3}$ ), and the same is ob served with the angular particles. So the M1 cement mix is more compact than the M2. A comparison can now be made between the densities of matrices with and without particles. The densities of those of the M1Sph and M1M mortars are very close to the den sity of the M1. On the other hand, the addition of particles to the M2 cement mix does seem to affect the porosity of the matrix. Thus, estimated values $n_{\mathrm{p}}$ of matrix porosity related to maximum values of matrix density have been deduced (Table 1) from Eq. (2):

$n_{\mathrm{p}} \frac{\rho_{\mathrm{M}}^{\max } \rho_{\mathrm{M}}}{\rho_{\mathrm{M}}^{\max }}$,

in which $\rho_{\mathrm{M}}^{\max }$ is the maximum density of the reference $\left(2.27 \mathrm{~g} / \mathrm{cm}^{3}\right.$ for matrix M1 and $2.30 \mathrm{~g} / \mathrm{cm}^{3}$ for matrix M2L) and $\rho_{\mathrm{M}}$ is the calcu lated value obtained from Eq. (1). The density of the matrix with small angular particles M2S $\left(2.06 \mathrm{~g} / \mathrm{cm}^{3}\right)$ is lower than that of mor tars with medium or large particles M2M and M2L (2.19 and $2.30 \mathrm{~g} /$ $\mathrm{cm}^{3}$, respectively). So the larger the particles, the higher the density of the matrix and the lower its porosity: the small particles surely hinder the escape of bubbles during the processing. Finally it is noticeable that the matrix of the M2M concrete, with medium sized particles, is similar in porosity and density to that of the M2 (with out particles) (Table 1 ).

\section{Bending tests}

\subsection{Specimen geometry and experimental set up}

Each type of composite was subjected to twenty bending tests to determine the Weibull characteristics of the different mixes and to measure each Young modulus (a total number of $200 \mathrm{spec}$ imens was tested). The specimens were rectangular parallelepi peds of height $h=20 \mathrm{~mm}$, width $w=15 \mathrm{~mm}$ and length $L=$ $100 \mathrm{~mm}$. The length between supports was $L_{\mathrm{s}}=80 \mathrm{~mm}$, which gives a $L_{\mathrm{s}} / h$ aspect ratio of 4 , close to value used in other studies of cement bending tests $[30,31]$ and sufficient to guarantee pre dominance of bending internal stresses. These dimensions allow obtaining the level of ultimate strength for an effective volume about one hundred cube millimetre. A similar value of effective volume was used to study the fragmentation process of an ultra high strength concrete submitted to edge on impact tests [32]. The $h, w, L$ dimensions are well above the size of the small and medium sized ceramic particles but the particles of the M2L con crete are between 5 and $10 \mathrm{~mm}$, rather close to the width of the specimens. The specimens were taken from concrete blocks 60 to $70 \mathrm{~mm}$ thick, the surface exposed to tensile stresses during the test being coincident with the symmetry plane of these blocks. Each face of the specimens was cut with a diamond disc and highly pol ished to offer a perfectly smooth surface. Some particles added to M1 were pulled out during this process, however, particularly from the type M1Sph (with a weaker matrix and spherical particles), and these extractions may have lowered slightly the bending strength of this concrete.

Two set ups were used in the course of the tests (Fig. 2) both with hinged supports below and linear above, the latter positioning the specimen. In the first set up (Fig. 2 left), the LVDT extensome ter sits on the frame of the machine, so the contact of the lower supports is added to the deformation of the specimen. This arrangement does not provide an accurate measurement of the deflection of the specimen under load, so to measure the Young modulus a second set up was designed in which the extensometer rests directly on the specimen and on the lower supports, short circuiting the contact deformation (Fig. 2 right). This set up was validated by aluminium alloy specimens loaded at the same stress level as that of the concrete. The measured Young modulus of alu minium alloy was $69 \mathrm{MPa}$ with a standard deviation of $1 \mathrm{GPa}$.

\subsection{Probabilistic approach to flexural failure}

The failure of the types of cement based composite used in this study is brittle. Moreover, several loading/unloading cycles were performed with several specimens of each grade to measure the Young modulus. No loss of stiffness has been recorded before fail ure. So an instable crack is thought to propagate in the specimen from the weakest defect of the specimen leading to its sudden fail ure. The random nature of the failure may be described by a Wei bull law [33 36], which gives the probability of failure $P_{\mathrm{f}}$ of a structure as function of the maximum tensile stress $\sigma_{\mathrm{m}}$ that exists in the structure before failure:

$P_{\mathrm{f}} \quad 1 \quad \exp \left(V_{\text {eff }} \lambda_{0}\left(\frac{\sigma_{\mathrm{m}}}{\sigma_{0}}\right)^{m}\right)$

in which $\sigma_{0}$ and $\lambda_{0}$ are, respectively, the reference tensile stress and the reference density of critical defects, $m$ the Weibull modulus, $\lambda_{0} /$ $\left(\sigma_{0}\right)^{m}$ is the second Weibull parameter and $V_{\text {eff }}$ is the effective vol ume, i.e., the volume of the structure if the state of stress is uniform [36]:

$V_{\text {eff }} \quad \int_{\Omega}\left[\frac{\left\langle\sigma_{I}(x)\right\rangle}{\sigma_{\mathrm{m}}}\right]^{m} \mathrm{~d} \omega$
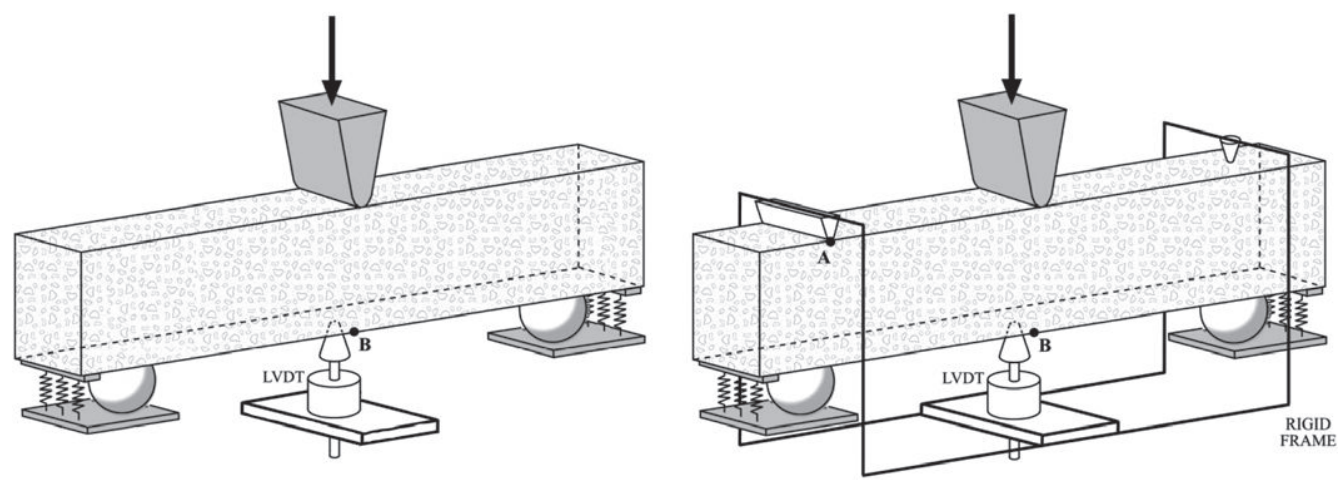

Fig. 2. Three-point bending test. Left: strength measurement set-up (Young modulus not measured). Right: Young modulus measurement set-up. 


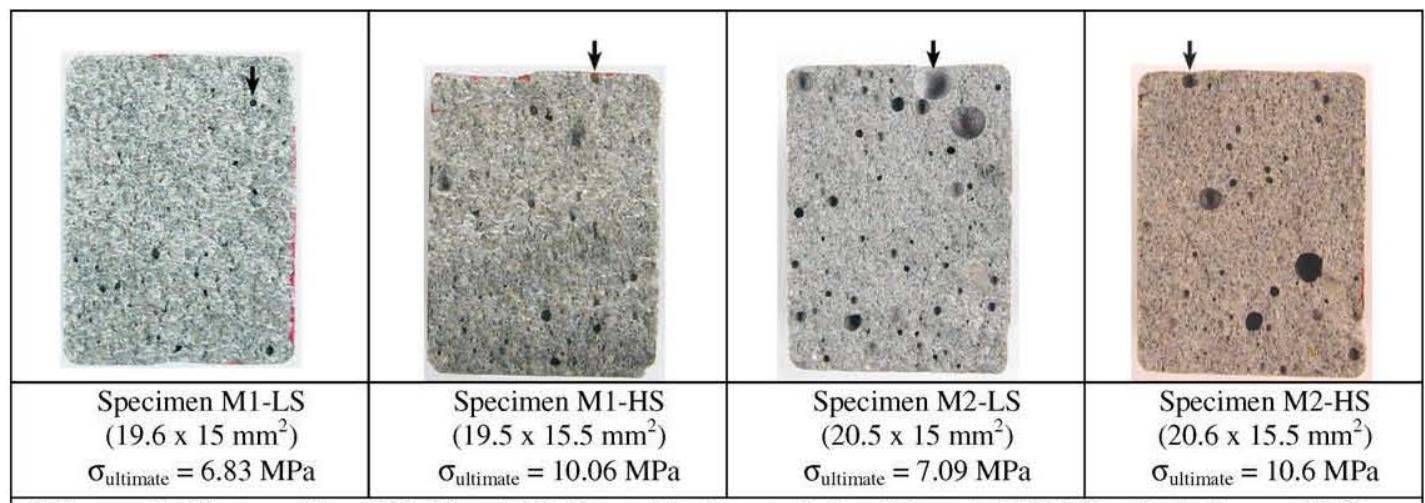

failure probably caused by: (M1-LS and HS: Pore with diameter below 0.5 mm); ( M2-LS: spherical pore of $2.7 \mathrm{~mm}$ diameter); (M2-HS: 1 mm diameter pore)

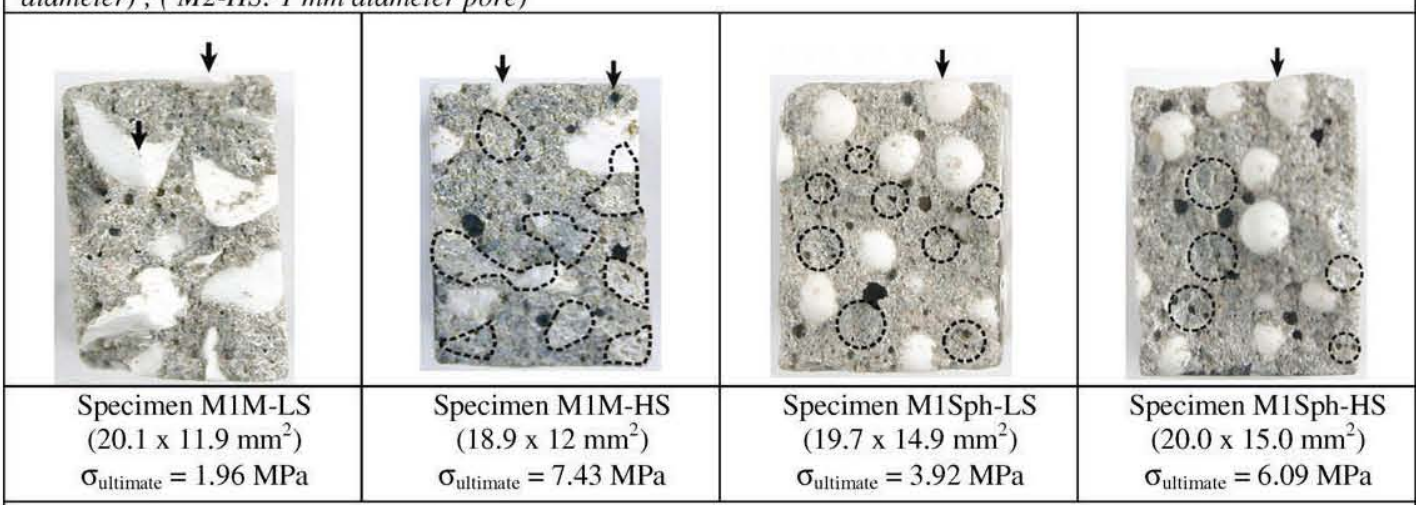

failure probably caused by: $L S, H S$ : Decohesion at particle/matrix interface: the particles act as defects

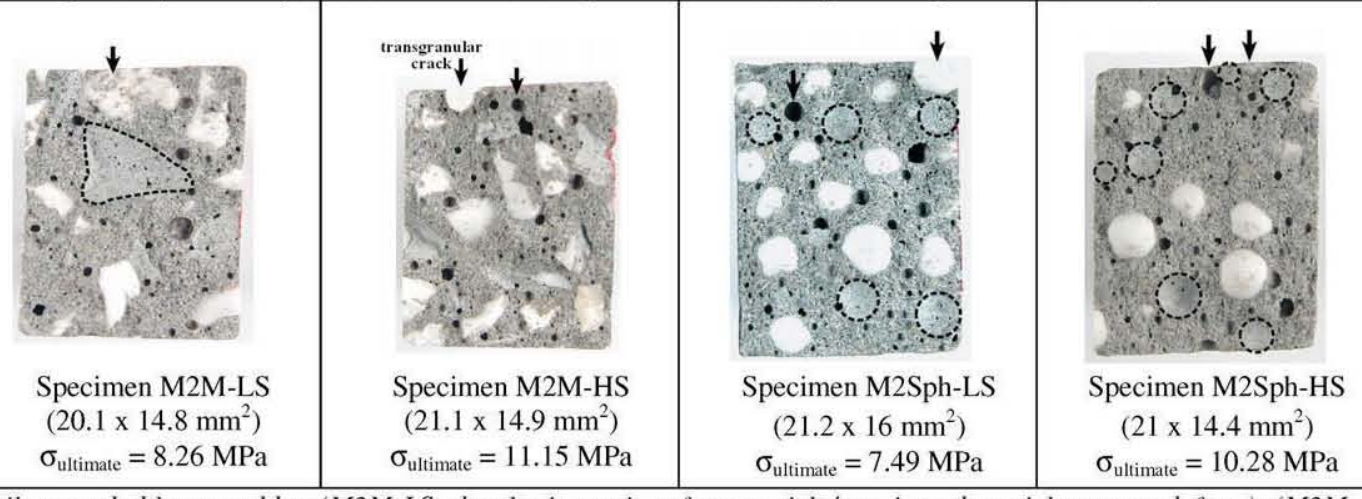

failure probably caused by: (M2M-LS: decohesion at interface particle/matrix and particles act as defects); (M2M$H S$ : pore of $1 \mathrm{~mm}$ and marked transgranular fracture indicating a good cohesion particle/matrix); (M2Sph-LS, HS: decohesion at the interface particle/matrix or pore of $1 \mathrm{~mm}$ diameter)

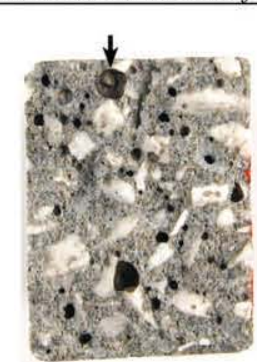

Specimen M2S-LS

$\left(19.9 \times 15 \mathrm{~mm}^{2}\right)$

$\sigma_{\text {ultime }}=9.17 \mathrm{MPa}$

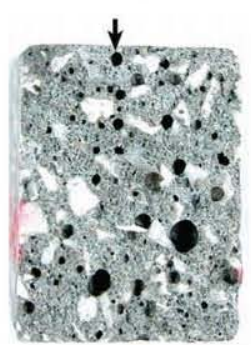

Specimen M2S-HS $\left(19.9 \times 15.1 \mathrm{~mm}^{2}\right)$

$\sigma_{\text {ultime }}=11.7 \mathrm{MPa}$

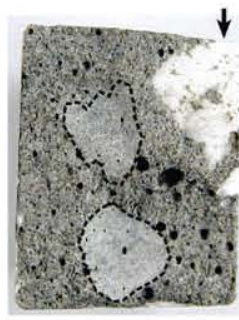

Specimen M2L-LS $\left(19.9 \times 15.9 \mathrm{~mm}^{2}\right)$ $\sigma_{\text {ultimate }}=7.18 \mathrm{MPa}$

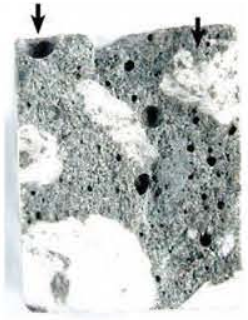

Specimen M2L-HS $\left(19.7 \times 15.6 \mathrm{~mm}^{2}\right)$ $\sigma_{\text {ultimate }}=11.8 \mathrm{MPa}$

failure probably caused by: (M2S-LS: pore of $2 \mathrm{~mm}$ diameter); (M2S-HS: pore of $0.7 \mathrm{~mm}$ diameter $) ;(M 2 L-L S$ : decohesion at the interface particle/matrix-particle of $6.7 \mathrm{~mm}) ;(M 2 L-H S$ : decohesion at the interface particle/matrix-particle of $5.7 \mathrm{~mm}$-or pore of $2.7 \mathrm{~mm}$ diameter)

Fig. 3. Fractured surfaces of M1, M2, M1M, M1Sph, M2M, M2Sph, M2S and M2L composite specimens after bending tests. Decohesion is shown with dotted lines. 
with $\sigma_{\mathrm{I}}(x)$ the maximum principal stress at a point $x$ and $\langle 0\rangle$ the po sitive part. The average failure stress $\sigma_{\mathrm{w}}$ is given by

$\sigma_{\mathrm{w}} \quad \sigma_{0}\left(V_{\mathrm{eff}} \lambda_{0}\right)^{\frac{1}{m}} \Gamma\left(\frac{m+1}{m}\right)$

in which $\Gamma$ is the second type eulerian function. Thus, the standard deviation $s_{\mathrm{d}}$ is given by

$s_{\mathrm{d}}^{2} \quad \sigma_{0}^{2}\left(V_{\mathrm{eff}} \lambda_{0}\right)^{\frac{2}{m}} \Gamma\left(\frac{m+2}{m}\right) \quad\left(\sigma_{\mathrm{w}}\right)^{2}$

The Weibull parameters can be identified from three point bending tests, for which the effective volume is given by

$V_{\text {eff }} \frac{V}{2(m+1)^{2}}$

The effective volume is seen to be strongly dependent on the Weibull modulus $m$ and much lower than the loaded volume $V$ of the specimen in a bending test. The Weibull modulus indicates whether the behaviour is probabilistic ( $m$ low) or deterministic ( $m$ high).

Table 2

Properties of the cement-based composites after three-point bending tests

\begin{tabular}{|c|c|c|c|c|c|c|}
\hline & & & M1 & M2 & \multicolumn{2}{|c|}{ M2 wet } \\
\hline \multicolumn{3}{|l|}{ Young modulus [GPa] $(E)$} & 33.0 & 34.0 & \multicolumn{2}{|l|}{ - } \\
\hline \multicolumn{3}{|l|}{ Average strength [MPa] $\left(\sigma_{\mathrm{w}}\right)$} & 8.48 & 8.9 & \multicolumn{2}{|c|}{8.99} \\
\hline \multicolumn{3}{|c|}{ Standard deviation strength [MPa] $\left(s_{\mathrm{d}}\right)$} & 0.72 & 0.81 & \multicolumn{2}{|c|}{0.89} \\
\hline \multicolumn{3}{|c|}{ Weibull modulus $(m)$} & 12.24 & 10.2 & \multicolumn{2}{|c|}{12} \\
\hline \multicolumn{3}{|c|}{ Effective volume $\left[\mathrm{mm}^{3}\right]\left(V_{\text {eff }}\right)$} & 66.8 & 95.7 & \multirow{2}{*}{\multicolumn{2}{|c|}{$\begin{array}{l}56.8 \\
8.58\end{array}$}} \\
\hline \multicolumn{3}{|c|}{ Average strength [MPa] $\left(\sigma_{\mathrm{w}}\right.$ for $\left.V_{\mathrm{eff}}=100 \mathrm{~mm}^{3}\right)$} & 8.21 & 8.86 & & \\
\hline & M1Sph & M1M & M2Sph & M2S & M2M & M2L \\
\hline Young modulus [GPa] $(E)$ & 47.0 & 44.9 & 48.0 & 46.0 & 47.5 & 49.8 \\
\hline Average strength [MPa] $\left(\sigma_{\mathrm{w}}\right)$ & 5.31 & 5.25 & 9.04 & 10.4 & 9.24 & 8.95 \\
\hline $\begin{array}{l}\text { Standard deviation strength } \\
{[\mathrm{MPa}]\left(s_{\mathrm{d}}\right)}\end{array}$ & 0.71 & 0.9 & 0.76 & 0.64 & 1.17 & 1.27 \\
\hline Weibull modulus $(\mathrm{m})$ & 8.6 & 5.9 & 13.6 & 17.7 & 8.8 & 8.26 \\
\hline Effective volume $\left[\mathrm{mm}^{3}\right]\left(V_{\text {eff }}\right)$ & 124 & 202 & 61.1 & 34.3 & 125 & 145 \\
\hline $\begin{array}{l}\text { Average strength [MPa] } \\
\quad\left(\sigma_{\mathrm{w}} \text { for } V_{\mathrm{eff}}=100 \mathrm{~mm}^{3}\right)\end{array}$ & 5.44 & 5.91 & 8.72 & 9.83 & 9.48 & 9.36 \\
\hline
\end{tabular}

\subsection{Results of the three point bending tests}

Fig. 3 shows some fractured surfaces of several cement compos ites reinforced with ceramic aggregates analysed, the upper face being the one in tension (lower face during testing). Among the large number of specimens tested for each mixture only two were selected for these figures, corresponding to a low (LS) and high (HS) flexural strength result.

\subsubsection{Mortar without particle reinforcement}

The results of the bending tests of the concretes are shown in Table 2. The three point bending tests with M2 were carried out on both dry and wet specimens (i.e., maintained in water $48 \mathrm{~h}$ ). The failure behaviour is very similar in the dry and wet specimens. The Weibull moduli are almost identical $(m \approx 10)$ and the average failure stress of the wet specimens is slightly lower. All the other concretes were tested dry. Cementitious matrix M1 shows less scatter of the failure load (higher Weibull modulus) and the aver age failure stress is slightly below that of the mortar with silica fume (M2). The absence of silica fume in the M1 specimens is par tially compensated by the smaller size of the pores in its matrix (see the fractured surfaces in Fig. 3). The elastic moduli are identi cal in all the types of mortar without particles ( $E \approx 34 \mathrm{GPa})$.

\subsubsection{Concrete type M1 with particle reinforcement}

The fractured surfaces of the two specimens of type M1M are shown in Fig. 3. The alumina particles or their hollows are seen as the white zones or the black dotted lines (Fig. 3). These fail ure patterns show a systematic decohesion (dotted lines) between the matrix and the particles. In addition, the projecting area of the surface corresponding to a particle/matrix decohesion is quite important (more than $40 \%$ of the surface, while the volumetric fraction of the particles is no more than $30 \%$ ). This shows that the crack spreads systematically across the particles/matrix inter face. This is an indication that the particles/matrix contacts are the 'weak' zones of the material. This assumption is confirmed by the fact that particles are always visible close to the upper part of the fractured surface. Failure is probably due to particle/matrix decohesion meaning that particles are acting as defects. This is confirmed by the Weibull parameters of the M1M in Table 2; the

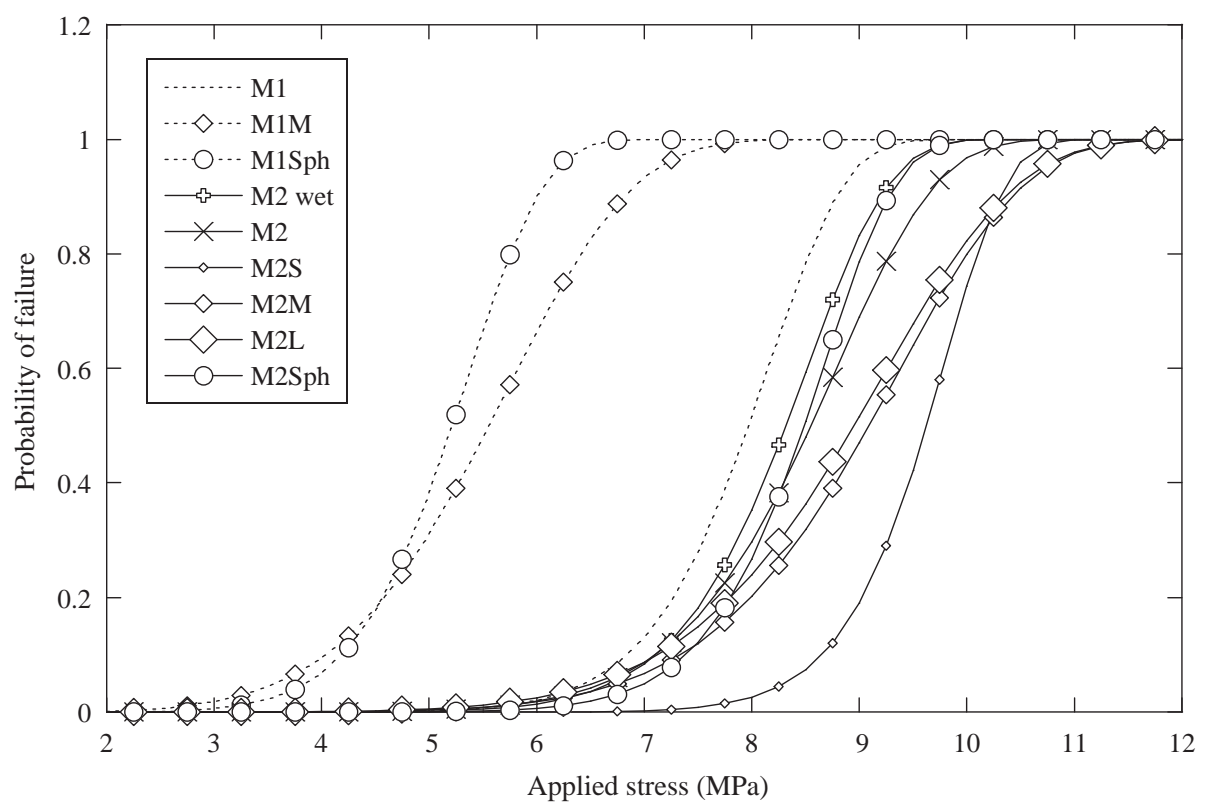

Fig. 4. Probability of failure versus applied stress in an effective volume of $100 \mathrm{~mm}^{3}$. 
flexural strength (5.25 MPa) is well below that in the M1 specimen $(8.48 \mathrm{MPa})$ and the scatter is much greater $(m=5.9)$. Only a failure provoked by particle/matrix decohesion can account for these parameters, since the very slight porosity would not induce such a loss of strength. For example, specimen M1M LS has no porosity in the upper zone (Fig. 3). Table 2 also shows the Weibull param eters of the type M1Sph, the average failure stress being practically identical to that of the M1M concrete, which confirms the hypoth esis of a failure provoked by particle/matrix decohesion, seemingly a threshold stress. The scatter of these stresses is lower, however $(m=8.6)$. This may be explained by the homogeneity of the distri bution of particles in the M1Sph specimens (spherical particles, uniform distribution, and higher density of particles). The two frac tured surfaces (Fig. 3) show a uniform particle distribution and the presence of at least two particles on the upper part of the picture.

\subsubsection{Concrete type $M 2$ with particle reinforcement}

The results of the bending tests with the M2 particulated spec imens are shown in Table 2. This time the average failure stress if particles are present (M2M, M2S, M2L) is slightly above that of the M2. So the question is whether the particles act as defects or as reinforcement. The clue lies with the fractured surfaces. The M2M LS specimen (that of the lowest strength in Fig. 3) has two large particles in the upper part. No porosity is visible near the loaded surface, so one of the two particles has probably triggered the failure. The surface of M2M HS shows no such decohesion. The only particle is broken across the grain, confirming the very good particle/matrix cohesion of the M2 mortar. In this case, two pores of $1 \mathrm{~mm}$ diameter may have initiated the failure. The M2Sph fractured surfaces (Fig. 3) show tiny pores and particle/ma trix decohesions, so the failure of these specimens may be due to either porosity or decohesion. However, the Weibull parameters indicate that the decohesion may have had a marked effect on the failure. As found in the M1Sph type, here the scatter of the fail ure stress is especially weak $(m=13.6)$. The spherical particles, uniform in their geometry, with a homogeneous distribution, form a uniform allocation of defects and explain the high Weibull mod ulus of this mix.

Twenty bending tests were done with the types M2S and M2L (small and large particles). Here again, the fractured surfaces clar ified the Weibull parameters (Fig. 3, Table 2). The fractured sur



Fig. 5. Stress-strain behaviour of composite specimens (M1, M1M, M1Sph) under uniaxial compression. faces of the M2S show a notable density of small pores, most probably trapped by small particles during the manufacture of the blocks. What is more, pore of $1.2 \mathrm{~mm}$ diameter is found near the fractured surface of M2S LS while the particles in this zone are of any size. It would seem, then, that it is porosity that origi nates failure, a possibility that is not refuted by the Weibull param eters. The high Weibull modulus $(m=17.7)$ is explained by the homogeneity of the population of pores and by their large number, and the particularly high failure level is due to their small size.

In contrast, the fractured surfaces of the M2L specimens (Fig. 3) show no porosity above $0.5 \mathrm{~mm}$ diameter. The failure originated from large particles visible in the tensile loaded zone. The average strength is the same as that of the M2Sph and M2M specimens, which suggests that the failure of the three types occurs nearly al ways when the interface stress reaches the cohesion strength be tween particles and matrix.

\subsection{Influence of the particle reinforcement on the strength of the cement based composites}

In the M1 matrices, the matrix/particle bond is very weak, so the particles behave as large size defects. This is why the failure stress is much lower in mortars containing particles. The Weibull modulus is weaker with angular particles, which act as defects of random size, shape and position in the specimens. With spherical particles there is less scatter as shown in Fig. 4 that illustrates the probability of failure versus the applied stress.

The behaviour of the M2 mortars is quite different. Particles may originate some of the failures in the M2Sph and M2M and most of those in the M2L specimens, even though the average fail ure stress is not reduced. The explanation lies in the smaller size of the pores in a mortar with particles. The M2L concrete has practi cally no pores of more than $1 \mathrm{~mm}$. When the particles are small (type M2S), the specimens break from the pores. The particles af fect the type of failure of the specimens and also the porosity that may trigger the failure. This explains the uneven scatter among the types. At first sight, it may appear paradoxical that the reinforced mortar with the greatest amount of porosity (M2S) is of great strength and of slight scatter. These results indicate that strength of ceramic reinforced mortars is above all a question of size of de fect and dispersion is more a question of size distribution of defects

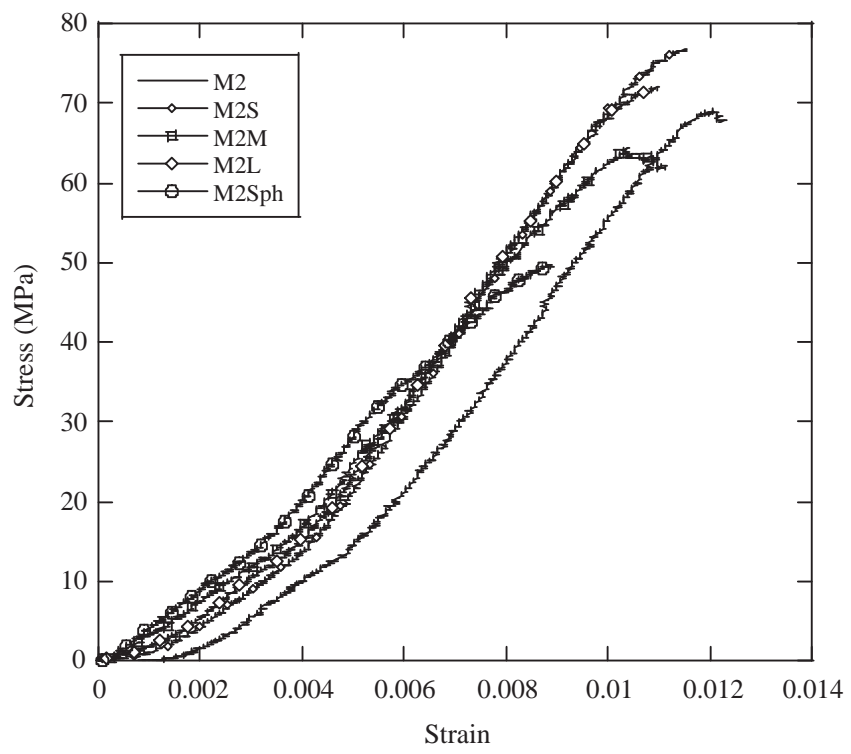

Fig. 6. Stress-strain behaviour of composite specimens (M2, M2S, M2L, M2Sph) under uniaxial compression. 
and they are absolutely not a question of amount of porosity (i.e. of volumetric fraction of pores).

\section{Uniaxial compression tests}

Simple compression tests were done to measure the strength of the different types of considered composites. This strength could then be compared to that of standard concretes. Since in this study the mortars contained particles of up to $10 \mathrm{~mm}$, we used cylindri cal specimens of $30 \mathrm{~mm}$ diameter and a height of $40 \mathrm{~mm}$ for the compression tests. Those dimensions give a height/diameter aspect ratio of 1.33 , close to value used in previous works for compression tests of cement materials $[10,37,38]$.

Table 3

Properties of cement-based composites after uniaxial compression tests

\begin{tabular}{lllllll}
\hline & & & M1 & & & M2 \\
\hline Min strength (MPa) & & & 40.8 & & & 63.5 \\
Max strength (MPa) & & & 70.6 & & & 71.2 \\
Mean strength (MPa) & & & 59.3 & & & 66.8 \\
& M1Sph & M1M & M2Sph & M2S & M2M & M2L \\
\hline Min strength (MPa) & 25.45 & 35.2 & 34.7 & 68.7 & 67.6 & 66.2 \\
Max strength (MPa) & 32.8 & 41.7 & 50.1 & 84.1 & 74.0 & 73.5 \\
Mean strength (MPa) & 29.1 & 38.3 & 42.4 & 76.0 & 70.8 & 69.85
\end{tabular}

\subsection{Specimens manufacture and experimental set up}

A hydraulic machine with a load capacity of $1000 \mathrm{kN}$ was used for the tests. Four cylindrical specimens were prepared of each type of material. They were diamond drilled from blocks of 60 to $70 \mathrm{~mm}$ thickness and the cylindrical surface was polished to elim inate any roughness or defects caused by the drilling. Cylindrical samples were cut from cement blocks with a diamond cuter. The end surfaces were then cut, rectified and polished of mode than the lack of parallelism between surfaces was around 5/100 mm and not greater than $1 / 10 \mathrm{~mm}$. The compression set up was equipped with two rigid compression plates, the upper plate linked by a ball and socket joint to the top bar. This joint maintains the uniaxial load on the specimen even if a defect should arise in the parallelism of the flat surfaces. The results of tests showed an elas tic brittle behaviour (representative curves are presented for all composite types in Figs. 5 and 6).

\subsection{Results of the uniaxial compression tests}

\subsubsection{Mortar without particle reinforcement}

Table 3 shows the failure stresses under simple compression of the cement based materials. Fig. 5 illustrates the state of damage of mortars without particles after their failure: multiple cracks, ax ial or slightly inclined. Most of these cracks pass through pores in

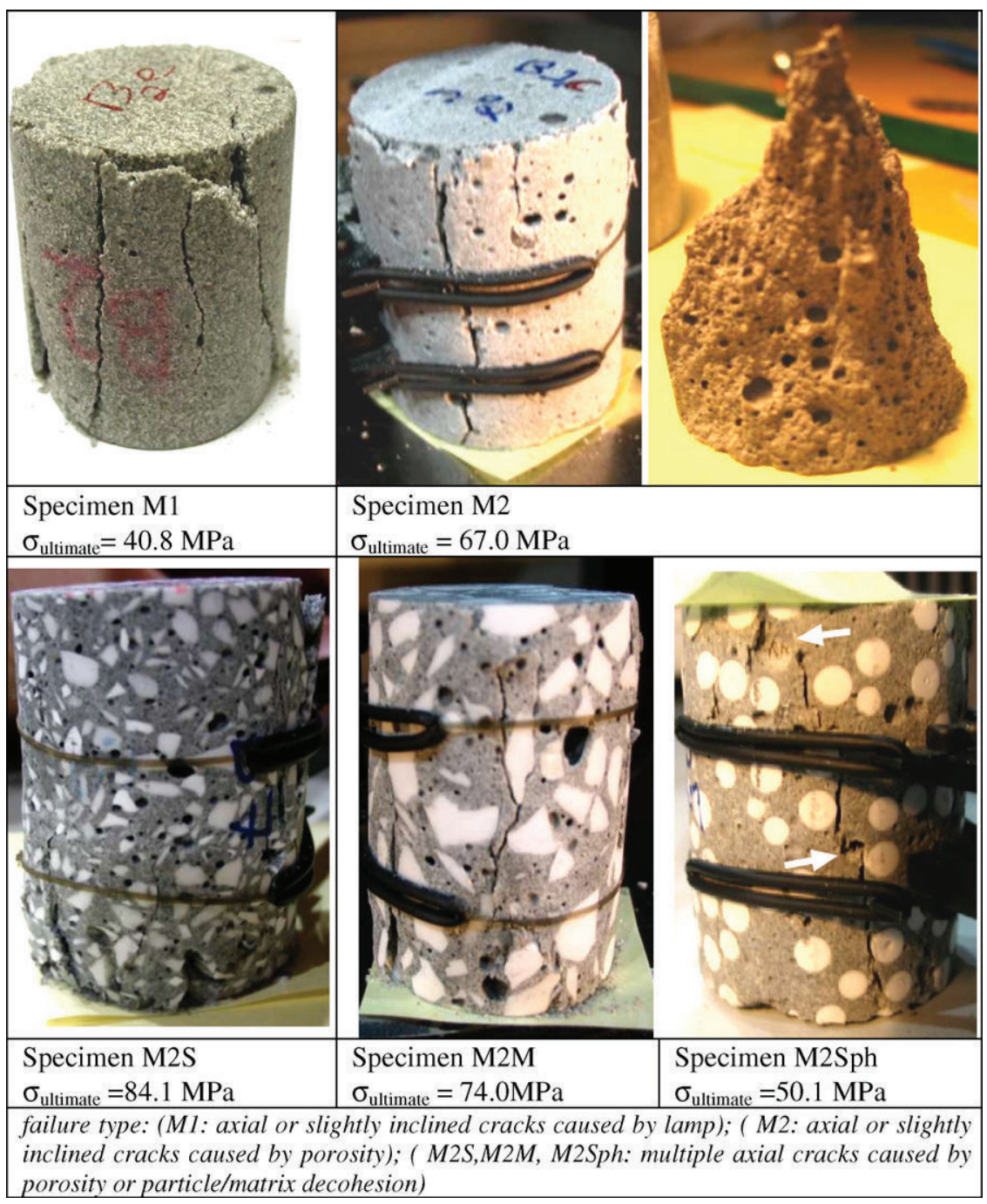

Fig. 7. Composite specimens after failure under uniaxial compression. 


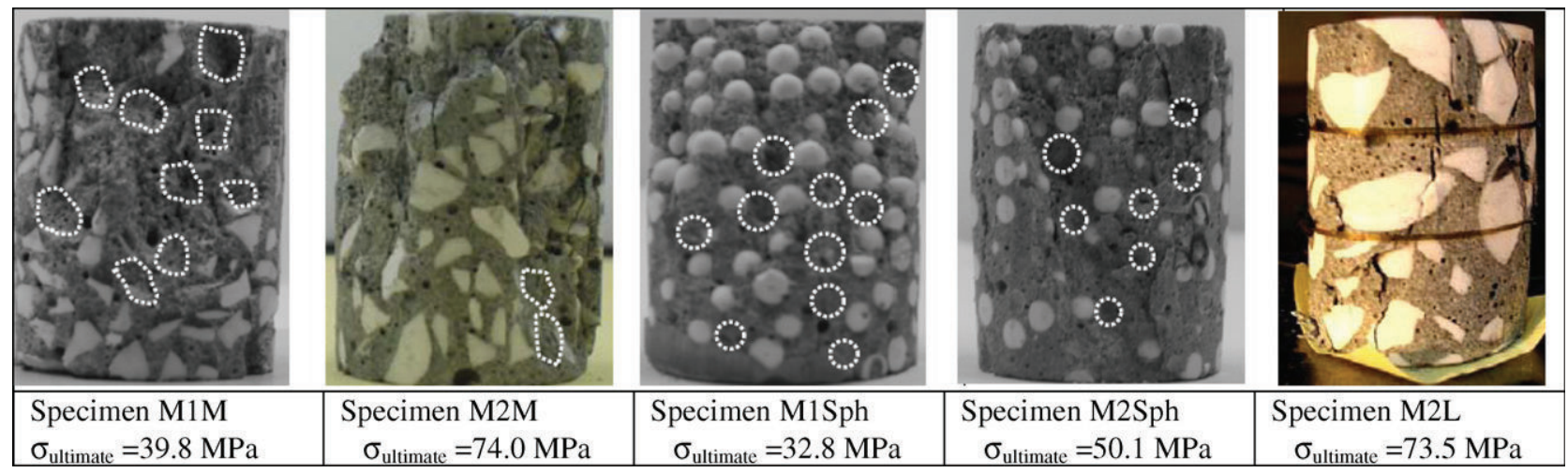

Fig. 8. Fracture surfaces of composite specimens (M1M, M2M, M1Sph, M2Sph) after failure under uniaxial compression. Decohesion is showed with dotted lines.

the material. Specimen M2, for example, has a cone of breakage with its base at the edge of the cylindrical specimen. The cone shows abundant porosity and the cracks originate in these round defects. In fact, pores are seen to be cut horizontally rather than vertically (Fig. 7). These observations show that pores are sites of initiation of the cracks. Multiple cracking initiates from the largest porosities and leads to the total ruin of the specimens.

The lowest strength of the M1 specimens (40.8 MPa) is ex plained by the presence of lump, visible on the fractured surface The results show a fairly clear greater strength of the mortars with silica fume. And even if the macroscopic porosity of the M1 mor tars is lower, its strength is below that of the M2 mortars.

\subsubsection{Concrete type M1 with particle reinforcement}

As observed in bending, the strength of the concretes with either angular or spherical particles and without silica fume matrix is clearly below that of the M1 mortar (Table 3). The strength of the $\mathrm{M} 1 \mathrm{M}$ is notably diminished and that of the M1Sph is only half that of the M1. After failure, the specimens have a large number of cracks influenced by the presence of the alumina particles, although the angular particles seem to hinder the propagation of cracks more than the spherical particles. Failure is probably a con sequence of the particle/matrix decohesion (Fig. 8), as in the case of bending even if the precise cause of failure is not known under simple compression.

\subsubsection{Concrete type $M 2$ with particle reinforcement}

Two to four tests were done with M2 mortars with particles. The fractured surfaces of specimens M2M and M2Sph show numerous mainly axial cracks. A number of particle/matrix deco hesions of the M2M material may have affected the final failure whereas a part of the cracks of the M2Sph specimen seem to orig inate in pores within the material (white arrows in Fig. 7). Here again, as in 3 point bending, the average strength of the M2M is above that of the M2 without particles. The rating is again similar to that of flexion. The strength of the M2Sph specimens is mark edly lower; the upper white arrow in Fig. 7 suggests that a pull out of the spherical particles occurred during the compression loading, which may have weakened the M2Sph specimens.

Table 3 shows the resistance under uniaxial compression of M2S and M2L composites. The strength of the M2 mortars with angular particles seems more or less identical, but the M2S has more porosity and the M2L contains particles of around $1 \mathrm{~cm}$. The post mortem analysis confirm that failure may have been originated in the pores of $\mathrm{M} 2 \mathrm{~S}$ and in the particle/matrix decohe sion in M2L. As in bending, the number of pores in M2S is

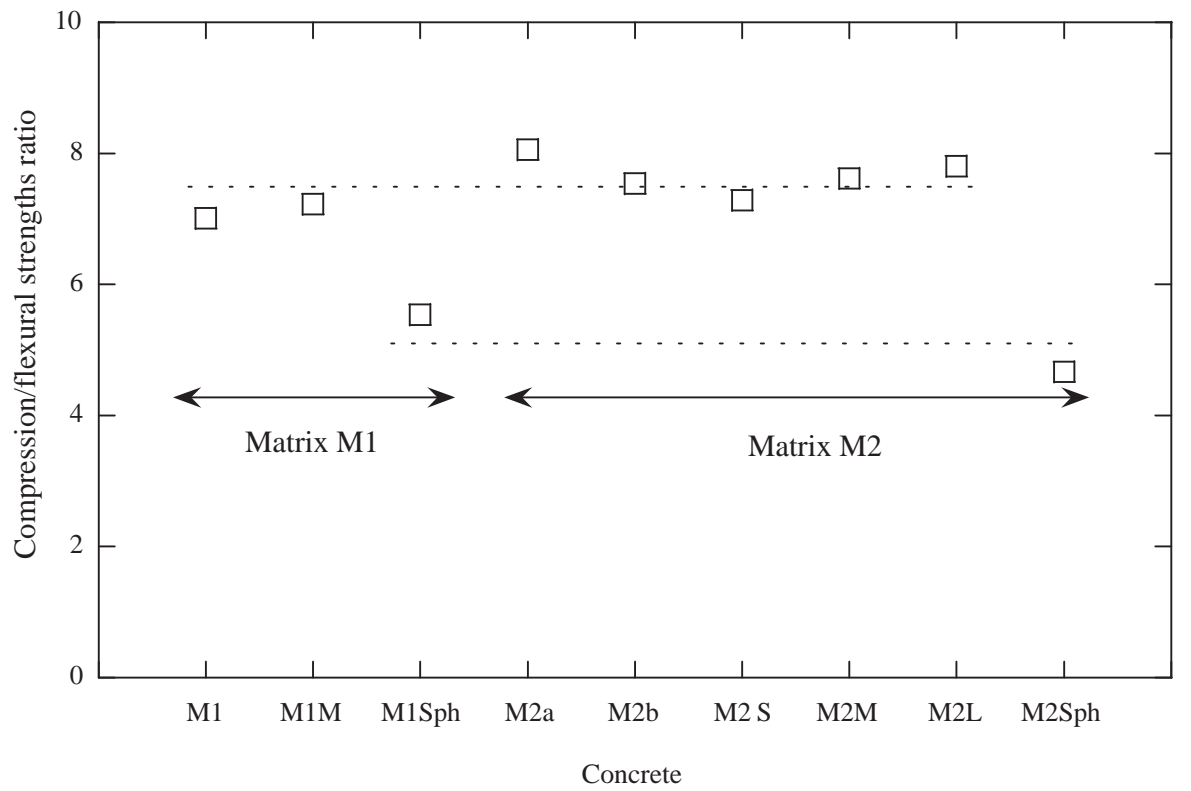

Fig. 9. Ratio of compression/flexural strengths of the set of cement-based composites with and without particle-reinforcement. 
compensated by their small size. The high strength of the M2L also shows good particle/matrix cohesion in M2 matrices. It is suf ficient to promote few transgranular fractures of some alumina particles even if cracks are generally going round the large parti cles (Fig. 8).

\subsection{Summary of the role of microstructure in the resistance under uniaxial compression}

Despite the reduce number of compression tests performed for each materials, some conclusions may be drawn. The addition of silica fume is seen to have a beneficial effect on the resistance of cement based composites to uniaxial compression, particularly if mortars contain particles, in spite of the slight increase of the mil limetric porosity. Matrices without silica fume have much less par ticle/matrix cohesion, and this leads to poor resistance of the M1 matrices with any type of particles in comparison with the M2 par ticulate concretes. This is consistent with the beneficial effect of silica fume observed by Caliskan [17] on the strength and density of interface between the matrix and aggregates. A comparison be tween the uniaxial compression strength of M2 concretes rein forced with angular particles (M2S, M2M, M2L) does not show a significant effect of their size, regards to the deviation of failure stresses of these materials. However, the strength is clearly dimin ished when particles are spherical. A pull out of the spherical par ticles during the unconfined compression loading may have weakened the M1Sph and M2Sph specimens.

There is a notable coherence between the results of the com pression and the bending tests. The diagram in Fig. 9 shows the ra tio between compression and flexural strength for each type of cement based composites with and without particle reinforce ment. This ratio has a value close to 7.5 for the concretes with angular particles. For spherical particles the ratio decreases to 5.0 , which is consistent with the change in failure initiation mech anism suggested previously.

\section{Conclusions}

The behaviour of both cement based composites with and with out particle reinforcement is shown by three point bending and uniaxial compression tests to be elastic brittle. We found that the mode of failure is the result of competition between two types of defects in the concrete: on one hand the pores and on the other ceramic inclusions when these act as defects. In mortars without silica fume, adhesion between alumina particles and the matrix is weak and the particle/matrix interface will trigger the failure. Alternatively, when silica fume is added to the cement paste, adhe sion between particles and the matrix is raised (especially with angular particles) and failure will be originated from the pores when small or medium particles are used or from a particle/matrix decohesion when medium and large particles are used. The shape of particles may also influence the strength of cement based com posites since the spherical ones lead more frequently to failure in comparison with angular particles of similar size. The scatter of strengths, and therefore the probabilistic (with low Weibull mod ulus) or deterministic (with a high modulus) behaviour of con cretes, reflects the scatter of the sizes of the defects, be they pores or particles. Concretes with pores of uniform size (observed with small particles) and/or with a granulometric distribution of the alumina particles around a single size (spherical particles) show less scatter among the levels of strength. To conclude, the combination of a statistical approach of failure and post mortem observations appears to be essential to analyse and understand the role of microstructure in the quasi static strength of this type of composites.

\section{Acknowledgements}

The authors are indebted to the Spanish Comisión Interministe rial de Ciencia y Tecnología (Project MAT2002 03339) and to the Comunidad Autónoma de Madrid (CCG06 UC3M/DPI 0796) for the financial support of this work and to the Délégation Générale pour l'Armement (DGA/France) for the mobility grant provided to Dr. Forquin.

\section{References}

[1] den Reijer PC. Impact on ceramic faced armours. PhD thesis. Delf University of Technology; 1991.

[2] Zaera R, Sánchez-Gálvez V. Analytical modelling of normal and oblique ballistic impact on ceramic/metal lightweight amours. Int J Impact Eng 1998;21(3):133-48

[3] Matchen B. Applications of ceramics in armor products. Key Eng Mater 1996;122:333-42.

[4] Riou P, Beylat L, Cottenot C, Derep JL. Impact damage on silicon carbide: first results. J Phys IV 1994;4:281-7.

[5] Forquin P, Tran L, Louvigné PF, Rota L, Hild F. Effect of aluminum reinforcement on the dynamic fragmentation of $\mathrm{SiC}$ ceramics. Int J Impact Eng 2003;28(10):1061-76.

[6] Jovicic J, Zavaliangos A, Ko F. Modeling of the ballistic behavior of gradient design composite armors. Compos Part A 2000;31:773-84.

[7] Arias A, Zaera R, López-Puente J, Navarro C. Numerical modeling of the impact behavior of new particulate-loaded composite materials. Compos Struct 2004;61(1-2):151-9.

[8] Arias A, Zaera R, López-Puente J, Navarro C. Manufacturing and compressive behaviour of a polymeric material loaded with ceramic particles. Boletín de la Sociedad Española de Cerámica y Vidrio 2004;43(2):401-5.

[9] Zaera R, Arias A, Navarro C. An engineering model on penetration of eroding rods into ceramic/polymer composite. J Phys IV 2003;110:609-14.

[10] Forquin P, Arias A, Zaera R. An experimental method of measuring the confined compression strength of high-performance concretes to analyse their ballistic behaviour. J Phys IV 2006;134(1):629-34.

[11] Duval R, Kadri E. Influence of silica fume on the workability and the compressive strength of high-performance concretes. Cem Concr Res 1998;28(4):533-47.

[12] Prokopski G, Langier B. Effect of water/cement ratio and silica fume addition on the fracture toughness and morphology of fractured surfaces of gravel concretes. Cem Concr Res 2000;30:1427-33.

[13] Papadakis VG. Experimental investigation and theoretical modeling of silica fume activity in concrete. Cem Concr Res 1999;29:79-86.

[14] Kearsley EP, Wainwright PJ. The effect of porosity on the strength of foamed concrete. Cem Concr Res 2002;32:233-9.

[15] Bhanja S, Sengupta B. Influence of silica fume on the tensile strength of concrete. Cem Concr Res 2005;35:743-7.

[16] Atis CD, Ozcan F, Kilic A, Karahan O, Bilim C, Severcan MH. Influence of dry and wet curing conditions on compressive strength of silica fume concrete. Build Environ 2005;40(12):1678-83.

[17] Caliskan S. Aggregate/mortar interface: influence of silica fume at the microand macro-level. Cem Concr Compos 2003;25:557-64.

[18] Rao GA, Prasad BK. Influence of the roughness of aggregates surface on the interface bond strength. Cem Concr Res 2002;32:253-7.

[19] Li Hui, Xiao Hui-gang, Jie Yuan, Ou Jinping. Microstructure of cement mortar with nano-particles. Compos Part B 2004;35:185-9.

[20] Jo Byung-Wan, Kim Chang-Hyun, Tae Ghi-ho, Park Jong-Bin. Characteristics of cement mortar with nano-SiO 2 particles. Constr Build Mater 2007;21:1351-5.

[21] Cargile J, O'Neil F, Neeley B. Very-high-strength concretes for use in blast- and penetration resistant structures. The AMPTIAC Quart 2003;6(4):61-6.

[22] Cheyrezy M, Maret V, Frouin L. Microstructural analysis of RPC. Cem Concr Res 1995;25(7):1491-500.

[23] Li QM, Reid SR, Wen HM, Telford AR. Local impact effect on hard missiles on concrete targets. Int J Impact Eng 2005;32:224-84.

[24] Rosenberg Z, Yeshurun Y. The relation between ballistic efficiency and compressive strength of ceramic tiles. Int J Impact Eng 1998;7(3): 357-62.

[25] Forquin P, Denoual C, Cottenot CE, Hild F. Experiments and modelling of the compressive behaviour of two SiC ceramics. Mech Mater 2003;35: 987-1002.

[26] Forrestal MJ, Frew DJ, Hanchak SJ, Brar NS. Penetration of grout and concrete targets with ogive-nose steel projectiles. Int J Impact Eng 1996:18(5):465-76.

[27] Cortés R, Navarro C, Martinez MA, Rodriguez J, Sanchez-Galvez V. Numerical modelling of normal impact on ceramic composite armours. Int J Impact Eng 1992;12(4):639-50.

[28] Kjellsen KO, Atlassi EH. Pore structure of cement silica fume systems. Presence of hollow-shell pores. Cem Concr Res 1999;29:133-42.

[29] Zelic J, Krstulovic R, Tkalcec E, Krolo P. The properties of Portland cementlimestone-silica fume mortars. Cem Concr Res 2000;30:145-52.

[30] Berthelot JM, Diouf B, Picart P. Statistical three-dimensional investigation of the damage evolution in heterogeneous materials. Eng Fract Mech 2008;75:1431-50. 
[31] Giaccio G, Tobes JM, Zerbino R. Use of small beams to obtain design parameters of fibre reinforced concrete. Cem Concr Compos 2008;30: 297-306.

[32] Forquin P, Hild F. Dynamic fragmentation of an ultra-high strength concrete during edge-on impact tests. ASCE J Eng Mech 2008;134(4): 302-15.

[33] Weibull W. A statistical theory of the strength of materials. Roy Swed Inst Eng Res; 1939, Report 151

[34] Weibull W. A statistical distribution function of wide applicability. ASME J Appl Mech 1951;18(3):293-7.
[35] Hild F, Denoual C, Forquin P, Brajer X. On the probabilistic deterministic transition involved in a fragmentation process of brittle materials. Comput Struct 2003;81:1241-54

[36] Davies DGS. The statistical approach to engineering design in ceramics. In: Proceedings of Brit ceram soc, vol. 22; 1973. p. 429-52.

[37] Forquin F, Gary G, Gatuingt F. A testing technique for concrete confinement at high strain rate. Int J Impact Eng 2008;35:425-46.

[38] Lu Xiaobin, Hsu Cheng Thomas. Behaviour of high strength concrete with and without steel fiber reinforcement in triaxial compression. Cem Concr Res 2006;36:1679-85. 